\title{
Traumatic haemarthrosis of the knee - indication to acute arthroscopy
}

\author{
Bilik A, Krticka M, Kvasnicka P \\ Department of Traumatology, the Faculty Hospital Brno, Traumacentre, Brno, Czech Republic. \\ a.bilik@pobox.sk
}

\begin{abstract}
Background and aims: The most common mechanisms of the knee injuries are distorsions and contusions. Fairly often they will result in hemarthrosis. The purpose of this study was to evaluace the findings in patiens with acute traumatic hemarthrosis of the knee and based on these findings estimate the necessity of acute artroscopic examination with therapeutic procedur.

Material and methods: In the years 2007 and 2009 a total of 266 patients underwent acute arthroscopic examination in Department of Traumatology the Faculty Hospital Brno after the acute knee trauma. Patiens with other intraarticular bone fractures were excluded.

Results: The ruptures of the ACL were the most common findings (52\%), meniscal tear $(27 \%)$, dislocation of the patella (11\%) and chondral or subchondral fractures were (13\%). In 224 (84\%) cases was performed artroscopic therapeutic procedure and in only $43(16 \%)$ case was performed routine arthroscopic examination. Conclusions: Based on our findings we believe that arthroscopic examination is necessary in patiens with acute traumatic haemarthrosis of the knee and it is better methods like NMR examination because is provided to execute therapeutic procedure (Tab. 1, Fig. 2, Ref. 8). Full Text in PDF www.elis.sk.

Key words: haemarthrosis, knee joint, arthroscopy, acute injury.
\end{abstract}

Knee contusion and distorsion are the most frequent injury mechanisms in sporting and unsporting activities. Pain, swelling and tension sanguine knee exudate is rising in few hours after acute knee injury. Traumatic knee haemarthrosis presents diagnostic difficulty in acute injury stage, it limits knee investigation and it should be disabled because of the pain. Nowadays, patient with acute knee haemarthrosis should be definitely admitted on Orthopaedic or Trauma Surgery Department.

The most frequent knee haemartrosis causations (except X-ray verified intraarticular fractures) are: anterior cruciate ligament rupture, meniscus rupture, patella luxation, collateral medial ligament rupture, chondral or osteochondral fractures and capsular tears.

Nuclear magnetic resonance (NMR) opens new chances in acute knee injury diagnostic. Intraarticular or extraarticular knee lesions are not only NMR benefits. On the other side there is a problem of NMR availability and waiting period for this examination.

Arthroscopy (ASC) is gold standard diagnostic method for intraarticular knee injury. Naturally, ASC has disadvantages, such as invasivity (risk of surgery) and this method can not verify associated extraarticular injuries, which NMR can. The arthroscopy is method of choice in patients with acute knee haemarthrosis, regarding to its good availability and exact intraarticular injury

Department of Traumatology, the Faculty Hospital Brno, Traumacentre, Brno, Czech Republic

Address for correspondence: A. Bilik, MD, PhD, Department of Traumatology, the Faculty Hospital Brno, Jihlavska 20, CZ-625 00 Brno, Czech Republic.

Phone: +420.5 .32232662$ diagnostic possibilities in conjuction with clinical examination in general anaesthesia.

\section{Methods}

From January 2007 to December 2009267 injured knees were treated in 266 patients with acute knee haemarthrosis in our department. One patient had injured both knees. 121 patients underwent acute knee arthroscopy till 24 hours from injury, 87 patients till 48 hours and in 58 patients till 72 hours from injury. Patients with patella fractures, femoral or tibial condyl fractures except intercondylar eminence were excluded from this study.

151 men and 115 women were treated in our patient file. Mean age of our patients was 33 yeas (17-81 years). Out-patients with acute knee haemarthrosis were promptly admitted to the Trauma Surgery Department of the Faculty Hospital Brno. Every patient underwent standard knee X-ray examination in anteroposterior and lateral projection. Knee puncture was made in 166 patients (62\%) before admitting to hospital. Arthroscopy was made in all patients to 3 days at the latest. Clinical knee examination in general anaesthesia forgo all arthroscopies. In our study we have not performed NMR even in one patient before arthroscopy. Patients with excluded knee haemarthrosis were not fit in this study.

\section{Results}

Statistically average annual incidence of acute knee haemarthrosis is $4.7 \%$ on 10000 inhabitants. Sport injury was noted in 183 cases from total number of 267 injured knees and mechanism 


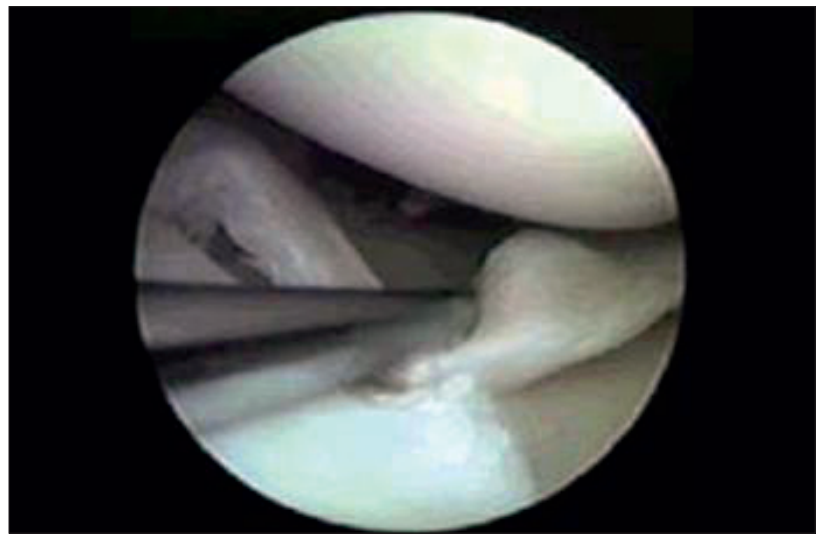

Fig. 1. Rupture of meniscus in red zone.

of injury was direct or distorsion. Knee joint was inured only in 29 cases during the motor vehicle accident. Football, skiing, tennis and squash were the most frequent sports, that were connected with knee injury and haemarthrosis. Anterior cruciate ligament (ACL) rupture was the most frequent find during the arthroscopy, it was in $52 \%$ of patients. Isolated ACL rupture was verified in 84 cases from total 139 ACL ruptures, 55 patients had combined injury. ACL rupture combined with collateral medial ligament (CML) rupture occurred in 23 cases, combination with meniscus rupture occurred in 19 cases and unhappy trias (ACL, CML and meniscus rupture) was identified in 13 cases. Isolated CML rupture was not verified. ACL arthroscopy treatment resulted from morphological ligament rupture find. ACL reinsertion was not used in any case. Non-interponing and non-fibrillated ruptured LCA was kept in situ for healing up. This procedure was applied in 52 cases. Complete ruptured LCA excision was applied in 87 cases. We made acute open suture in 34 cases of acute ruptured CML from total number of 36 cases. Only 2 ruptures of CML were I. grade, and they were kept for conservative treatment. Meniscus rupture was verified in 72 cases $(27 \%)$, isolated meniscus injury was found in 40 cases (Fig. 1). Meniscus ruptures combined with other injuries were found in remaining 32 ruptures. Rupture in white zone we found in 30 cases and partial excision of involved part was made. Rupture in red zone was noted in 42 cases, all these injury types were treated with acute suture (out side-in side technique, PDS fibre).

Tab. 1. The most common findings of 266 patients underwent acute arthroscopic examination.

\begin{tabular}{ll}
\hline Findings & Number of patients \\
\hline rupture of ACL & $139(52 \%)$ \\
isolated & 84 \\
ACL + CML & 23 \\
ACL + meniscus & 19 \\
ACL + CML + meniscus & 13 \\
\hline rupture of meniscus & $72(27 \%)$ \\
isolated & 40 \\
combined & 32 \\
\hline luxation of patella & $30(11 \%)$ \\
\hline osteochondral fracture & $35(13 \%)$ \\
\hline fracture of intercondyl. eminentia & $8(3 \%)$ \\
\hline
\end{tabular}

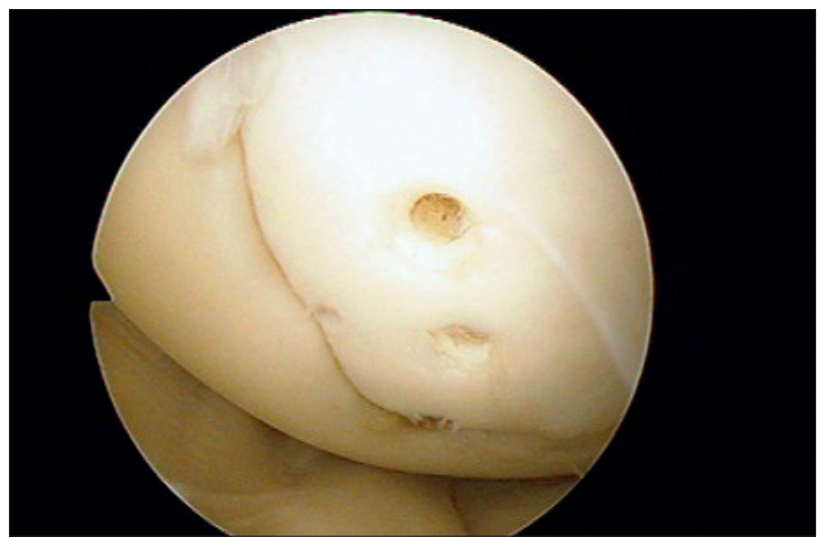

Fig. 2. Osteosynthesis of chondral fracture.

Medial meniscus was injured in 55 cases from total number of 72 meniscus rupture. Patella luxation was cause of 30 haemarthrosis $(11 \%)$. Lateral luxation was cause of all luxations. Standard anterolateral and proximal lateral port was used for visualisation of femoropatelar articulation. Only in favourable femoropatelar articulation is possible to made pure knee haematoma evacuation. It was made only in 4 cases from all our patients. Medial parapatelar ligament tear was found in 26 patients, we made acute suture with Vicryl fibre, method of transcutaneus stitch was made in 19 patients, method of open suture was made in 7 patients. Lateral patellar release was made in case of persisting lateral patellar dislocation. 20 patients underwent this procedure in our study.

Chondral and osteochondral fracture was identified in 35 cases $(13 \%)$. Patella was place of the most frequent occurance of osteochondral fractures or chondral avulsions (x16), medial femoral condyle (x9) and lateral femoral condyle (x10). 14 from 35 chondral fractures were connected with patella luxation, 7 with ACL rupture, 6 with meniscus rupture and 8 was isolated case. 9 patients were older then 50 years and they suffer from diferent gonarthrosis grade. Osteochondral fracture in high weighted zone had 35 patients, loose osteochondral fragment was fixed with mini-screws or resorbable screws in 12 cases (Fig. 2) or it was removed in 23 remaining patients. Intercondylic eminence fracture was grounds for ASC in 8 patients. Dislocated fracture (Meyers and McKeever type II and III) was solitar injury in all 8 patients. Technique of artroscopy assisted fracture reduction and reinsertion with 2 PDS stitches through 2 transtibial channeles (drilled through $\mathrm{C}$ targeting with $2 \mathrm{~mm}$ Kirschner wire) was used in all cases. The most frequent findings are sumarized in Table 1.

\section{Discussion}

Traumatic soft tissue knee injury, chondral and osteochondral fractures, its significance and status in joint degeneration have not been clerified to this day. Flexion and rotation movement or extension and rotation movement are the most frequent causations of the knee injury with haemarthrosis. These movements lead to injuries of soft intraarticular tissues (ligament and meniscus ruptures), but also to patella luxations. Above mentioned injury mechanism is 
also responsible for intercondylar eminence fractures (margin of intercondylic fossa is compressed to intercondylic eminence and it is breaking). Direct stroke is the second most frequent injury causation, which might cause cartilage avulsion or impaction. ASC is the best choice of diagnose and treatment for chondral and osteochondral fractures (1). In our study of patients with acute ASC (by 3 days from inury) for knee haemarthrosis we found bleeding origin in all cases (100\%). It was isolated LCA ruptures, menisci ruptures, chondral and osteochondral fractures, patella luxations or its combinations. Most patients in working age called for final treatment. We did not make LCA reconstruction in no case of LCA rupture, because of high risk of severe arthrofibrosis origination resulting in knee movement limitation (6). Complete injury mechanism anamnesis, $\mathrm{X}$-ray and clinical examination are sufficinet for diagnosis of patella luxation. However ASC can serve information about cartilage status and pertinent loose fragment (it may not be seen on X-ray) might be fixed or removed. Benefit of ASC in patients with patella dislocation would not be polemized with regard to information listed above. Loose osteochondral fragment we found in $46 \%$ patients $(16 / 35)$ with patella luxation. Similar findings are brought in literature (2).

Meniscus ruptures require operative treatment in most cases and treatment depend up the meniscus tear characteristics. Repair outcome is better in the event that suture is made in the earliest possible time from injury (7). The role of NMR in detecting meniscal ruptures that require operative treatment has been somewhat controversial (4). Abrupted intercondylar eminence is stabilized with resorbable material, it is sufficient and it does not require osteosynthetic material extraction (3). Arguments for and against arthroscopy or NMR realisation are constantly discussed in cases of acute knee haemarthrosis (5). NMR diagnostic importance should not been underestimated in relation to number of false positivity or negativity (8).

\section{Conclusion}

Acute knee injury with haemarthrosis is indication for arthroscopy. Bleeding origin visualization and possibility of injury treatment are arguments supporting arthroscopy indication. 267 knee arthroscopies were made in our study, in 244 cases ( $84 \%$ ) we found and treated cause of bleeding, in 42 cases ( $16 \%$ ) we made only diagnostic arthroscopy with knee joint flush. We suppose that blood in joint has a toxic effect to articular cartilage and it should be urgently evacuate, that is why arthroscopy has protective effect to cartilage. Therefore we indicate for acute surgery all patients as soon as possible regardless of the amount of blood in the knee joint. The timing of the procedure should be such that all possible lesions encountered can be treated in the same operation. We does not underestimate NMR diagnostic benefit, we recommend to make NMR before ASC in hospitals where is possibility to make it as a routine examination. But we use this method of investigation for the diagnosis of obscure chronic difficulties.

\section{References}

1. Kayaoglu EE, Binnet MS. Chondral and osteochondral fractures. Acta Orthop Traumatol Turc 2007; 41 (Suppl 2): 105-12.

2. Paša L, Kalandra S, Melichar I, Bilik A, Suchomel R. Arthroscopic management of traumatic luxation of the patella. Rozhl Chir 2006; 85 (10): 530-535.

3. Paša L, Višňa P, Kočiš J, Mužík V, Veselý R. Arthroscopic stabilization of the fractured intercondylar eminence. Acta Chir Orthop Traumatol Cech, 2005; 72 (3): 160- 63.

4. Sarimo J, Rantanen J, Heikkilä J, Helttula I, Hiltunen A, Orava S. Acute traumatic haemarthrosis of the knee. Is routine arthroscopic examination necessary? Scand J Surg 2002; 91: 361-364.

5. Sharef D, Banerjee A. Assessment and management of posttraumatic haemarthrosis of the knee. Br J Hosp Med (Lond) 2008; 69 (8): 459-460, $462-463$.

6. Shelbourne KD, Wilckens JH, Mollabashy A, DeCarlo M. Arthrofibrosis in acute anterior cruciate ligament reconstruction: The effect of timing of reconstruction and rehabilitation. Am J Sports Med 1991; 19: 332-336.

7. Venkatachalam S, Godsiff SP, Gardiny ML. Review of the clinical results of arthroscopic meniscal repair. Knee 2001; 8: 129-133.

8. von Engelhardt LV, Schmitz A, Pennekamp PH, Schild HH, Wirtz DC, von Falkenhausen F. Diagnostics of degenerative meniscal teras at 3- Tesla MRI compared to arthroscopy as reference standard. Arch Orthop Trauma Surg 2008; 128 (5): 451-456. 